\title{
Eggplant-Derived Biochar-Halloysite Nanocomposite as Supports of Pd Nanoparticles for the Catalytic Hydrogenation of Nitroarenes in the Presence of Cyclodextrin
}

Samahe Sadjadi ${ }^{1}$, Maryam Akbari $^{2}$, Bastien Léger $^{3}$, Eric Monflier $^{3}$, Majid M. Heravi $^{2}$

1- Gas Conversion Department, Faculty of Petrochemicals, Iran Polymer and Petrochemicals Institute, 15km Tehran-Karaj Highway, Tehran, 14977-13115, Iran

2- Department of Chemistry, School of Science, Alzhara University, Vanak Village Street, Tehran, 19938-91176, Iran

3- University of Artois, CNRS, Centrale Lille, ENSCL, University of Lille, UMR 8181, Unité de Catalyse et de Chimie du Solide (UCCS), Lens, F-62300, France

\section{Abstract}

A novel halloysite-hydrochar nanocomposite has been prepared and applied for the immobilization of Pd NPs to furnish an efficient catalyst for the hydrogenation of nitroarenes. It was confirmed that use of a catalytic amount of $\beta$-cyclodextrin ( $\beta-C D)$ could improve the yield of the reaction significantly. With the aim of investigation of the effect of combination of Hal and Char, Char surface modification, and the way of use of $\beta-\mathrm{CD}$ on the catalytic activity, several control catalysts were prepared and their catalytic activities were compared with that of the catalyst. It was confirmed that the use of Hal-Char as a support was more effective than the use of each component individually. Moreover, the use of $\beta-C D$ in its free form was more efficient than incorporating it to the framework of the catalyst or as a capping agent. It was also found that Char in its unmodified form was more efficient than modified ones. To justify the results, a precise study was carried out by comparing the 
Source: Sadjadi S, Akbari M, Léger B, Monflier E, Heravi MM. Eggplant-Derived Biochar-Halloysite Nanocomposite as Supports of Pd Nanoparticles for the Catalytic Hydrogenation of Nitroarenes in the Presence of Cyclodextrin. ACS Sustainable Chem Eng. 2019;7(7):6720-31. DOI: 10.1021/acssuschemeng.8b05992

average Pd particle size and loading of each samples. It was confirmed that the Pd particle size and dispersion effectively affected the catalytic activity. Additionally, $\beta-\mathrm{CD}$ amount was a key factor for achieving high catalytic activity.

Keywords: Halloysite, Biochar, Pd nanoparticles, Hydrogenation, Cyclodextrin 